\title{
Crapoulette: card craps with one deck
}

\section{DAVID K. NEAL and DUSTIN GENTILE}

The game of craps is one of the most popular casino games for players and spectators. Many people enjoy craps due to the party atmosphere it creates, and as many as twenty people can participate in a single round of the game. Anyone at the table can place a bet on any roll by the shooter. According to the Nevada Gaming Control Board Information Sheet [1], there were 400 craps tables open across the state of Nevada in 2013; only for Blackjack and Roulette were more tables licensed in the State.

Due to the popularity of craps, card craps was developed to simulate craps in states such as California where dice craps is illegal. In this game, a player uses multiple decks of cards that contain only the cards Ace, 2, 3, 4, 5, and 6, where an Ace holds the value of one. To start each game, there is a come-out roll where the shooter draws two cards to simulate the roll of two dice, then sums the values of the cards. The bets and rules for card craps are the same as in standard dice craps. Specifically, the shooter automatically wins if a sum of 7 or 11 is drawn, and automatically loses if a sum of 2,3, or 12 is drawn. Any other sum establishes a point. If a point is established, then the shooter continues to draw two cards at a time until either drawing a sum of 7 or the same point. Then the shooter wins if the point is drawn first and loses if a 7 is drawn first.

There does not appear to be one standard rule for drawing cards. Some venues use a shoe with 264 cards that are drawn without replacement and are replaced when about half the shoe has been used. Others use only 48 cards and replace the first two cards after a come-out roll if a point has been established. But then cards are drawn without replacement until the game is decided. Still other venues use a continuous shuffle machine where cards are replaced after each draw.

Card craps has become very popular in casinos across the United States due to its ease of playing and its inviting atmosphere. However it can be difficult to analyse mathematically due to the various methods of drawing cards with or without replacement. But variations of card craps can be designed that do not necessarily mimic dice craps, and that do have fixed methods of drawing cards which allow for easier mathematical analysis. To be marketable, any such game should give the House a slight advantage, should have ease and speed of play, and should allow onlookers to participate in bets.

In this paper, we shall describe a new type of card craps game that can be played in casinos or at home with friends. The game is played with one full deck of cards and we call it Crapoulette. Besides being fun to play, the game provides a way to illustrate several important probability concepts. In particular, we shall use the Law of Total Probability (the Partition Theorem, see [2, p. 14]) and the Law of Total Expectation (see [2, p. 34-35]) to derive the probability of the shooter winning and the average number of draws per 
game. We shall also derive the conditional average number of draws made among the shooter's winning games and losing games. Lastly, we describe how to determine the payoffs for various bets that could be made, and how to determine the House's average profit on a particular bet.

\section{The game}

In Crapoulette, the shooter draws one card from a standard shuffled deck of 52 cards, which is called the come-out roll. Any Jack, Queen, or King will be an automatic winner, while an Ace will be an automatic loser. If the shooter draws a 2 through a 10, then that value establishes a point. In this case, the shooter continues to draw one card at a time, without replacement, until drawing the same point or a Jack, Queen, King, or Ace. The shooter wins if the point or an Ace is drawn first, and loses if a Jack, Queen, or King is drawn first. These additional draws are called point draws.

A pass line bet is the general bet that the shooter wins. But there are various other side bets that can be made, such as the shooter winning on the come-out roll, the shooter winning after a point has been established, or the shooter winning after a point is established by redrawing the point. Because there are different probabilities of winning each bet, the House must offer different payoffs for each. The pass line is the most interesting bet to analyse which we now do.

We let $W$ be the event of winning a pass line bet, and let $X$ be the number of draws needed to complete a game. The game ends on one draw if a Jack, Queen, King, or Ace is chosen; thus, $\mathrm{P}(X=1)=\frac{4}{13}$ and $\mathrm{P}(X>1)=\frac{9}{13}$. The probability of initially drawing a Jack, Queen, or King is $\frac{3}{13}$, which gives the probability of winning on the first draw. Given that the game has ended on one draw, then three out of four times a Jack, Queen, or King has been drawn resulting in a win; thus, $\mathrm{P}(W \mid X=1)=\frac{3}{4}$. Therefore the probability of winning on the first draw is also given by

$$
\mathrm{P}(W \cap X=1)=\mathrm{P}(X=1) \times \mathrm{P}(W \mid X=1)=\frac{4}{13} \times \frac{3}{4}=\frac{3}{13} .
$$

We now consider the probability of the shooter winning given that a point has been established. Because a point having value 2 through 10 has been drawn, there are only three more of these values left in the deck along with 16 cards that are a Jack, Queen, King, or Ace. The drawing of one of these 19 cards now ends the game. The first card drawn is equally likely to be any of the 19 cards, 7 of which are 'winners'. Therefore the probability of winning given that a point has been established is $\frac{7}{19}$.

Now by the Law of Total Probability, the probability of the shooter winning is

$$
\begin{gathered}
\mathrm{P}(W)=\mathrm{P}(X=1) \times \mathrm{P}(W \mid X=1)+\mathrm{P}(X>1) \times \mathrm{P}(W \mid X>1) \\
=\frac{4}{13} \times \frac{3}{4}+\frac{9}{13} \times \frac{7}{19}=\frac{120}{247} .
\end{gathered}
$$


We summarise these results as:

Theorem 1: In the game of Crapoulette,

(a) the probability of winning on the first draw is $\frac{3}{13}$;

(b) the probability of winning after a point has been established is $\frac{7}{19}$;

(c) the probability of winning a pass line bet is $\frac{120}{247} \approx 0.48583$.

For comparison, the probability of winning a pass line bet in standard dice craps is $\frac{244}{495} \approx 0.49293$. And in American Roulette, where the wheel has numbers 1 to 36 plus two zeroes, the probability of winning a bet on each of a Red, Black, High, or Low number is $\frac{18}{38} \approx 0.47393$. Therefore, with 1:1 payoffs, a casino will make a greater average profit on Crapoulette than on craps, but not as great as with the roulette bets.

Now suppose $p$ is the probability of winning a bet, $q=1-p$ is the probability of losing, and there is a 1:1 payoff. Then, on average, for every $n$ bets of $\$ b$, the House earns $\$ b \times n q$ and pays out $\$ b \times n p$, which gives the House an average profit of $\$ b n(q-p)$. So for every 100 pass line bets of $\$ 5$ in Crapoulette, the average House profit is $\$ 500(127-120) / 247 \approx \$ 14.17$. On pass line bets in craps, the average profit would be $\$ 7.07$, while the average House profit for every 100 bets of $\$ 5$ on Red at the American Roulette wheel is about $\$ 26.32$.

\section{Other bets}

In addition to the pass line bet, various other bets can be made on the come out roll. For example one can bet on any single value being drawn, such as a 7. The probability of winning this bet is $p=\frac{1}{13}$, which make the odds against winning to be 12:1. In order for the House to make a profit, the actual payoff must then be less than 12:1 on this bet.

Indeed, if the payoff is $\$ a$ for a bet of $\$ 1$, then the average House profit per $n$ bets is $n(q-a p)$. In order for the House to profit, we must have $0<n(q-a p)$, which gives $a<\frac{q}{p}$, where the ratio $\frac{q}{p}$ gives the true odds against winning. So for $p=\frac{1}{13}$ and $q=\frac{12}{13}$, the House must use a payoff less than 12:1 in order to average a profit on the single number bet.

Another possibility is a bet on a row of three values (see Table 1), so that $p=\frac{3}{13}$ giving odds against winning of 10:3. The House could then use a 3:1 payoff. One could also bet that no point will be established, i.e. that the first draw will be a Jack, Queen, King, or Ace. In this case, the odds against winning are 9:4, so a 2:1 payoff would be appropriate. Table 1 shows a variety of bets that could be made on the come-out roll. 


$\begin{array}{cccc}\text { Bet } & \text { Prob. of win } & \text { Odds against } & \text { Payoff } \\ \text { Single number/Loss } & \frac{1}{13} & 12: 1 & 11: 1 \\ \text { Pair } & \frac{2}{13} & 11: 2 & 5: 1 \\ \text { Triple (row)/Win } & \frac{3}{13} & 10: 3 & 3: 1 \\ \text { Quadruple (column) } & \frac{4}{13} & 9: 4 & 2: 1 \\ \text { No point } & \frac{4}{13} & 9: 4 & 2: 1 \\ \text { Low }(2-7) & \frac{6}{13} & 7: 6 & 1: 1 \\ \text { High }(8-\text { K) } & \frac{6}{13} & 7: 6 & 1: 1 \\ \text { Pass line } & \frac{120}{247} & 127: 120 & 1: 1 \\ \text { Point } & \frac{9}{13} & 4: 9 & 2: 5\end{array}$

TABLE 1: Come-out bets

If a point is established, then further bets can be made at any time before each successive draw. At this time, the probability of the shooter winning the initial pass line bet is $7 / 19$ giving odds against winning of $12: 7 \approx 1.7: 1$. So the House could offer a payoff of say $1.5: 1$ for betting on the shooter to win after a point is established. This bet can be made after any draw that does not conclude the game because drawing any irrelevant card does not affect the probability of the shooter winning the game.

On point rolls, the odds against the shooter losing are $7: 12 \approx 0.58: 1$. Thus the House could give a 0.5:1 payoff for bets on the shooter to lose after a point has been established. Moreover, the probability that the point is redrawn first is $\frac{3}{19}$, and there is probability $\frac{4}{19}$ of first drawing each of the values Jack, Queen, King, and Ace. Therefore point roll bets can be made on these events as well. Table 2 shows various bets that be made before each point roll, and Figure 1 shows a possible betting table.

$\begin{array}{cccc}\text { Bet } & \text { Prob. of win } & \text { Odds against } & \text { Payoff } \\ \text { Point } & \frac{3}{19} & 16: 3 & 5: 1 \\ \text { Ace } & \frac{4}{19} & 15: 4 & 3.5: 1 \\ \text { Jack } & \frac{4}{19} & 15: 4 & 3.5: 1 \\ \text { Queen } & \frac{4}{19} & 15: 4 & 3.5: 1 \\ \text { King } & \frac{4}{19} & 15: 4 & 3.5: 1 \\ \text { Win } & \frac{7}{19} & 12: 7 & 1.5: 1 \\ \text { Loss } & \frac{12}{19} & 7: 12 & 0.5: 1\end{array}$

TABLE 2: Point roll bets 


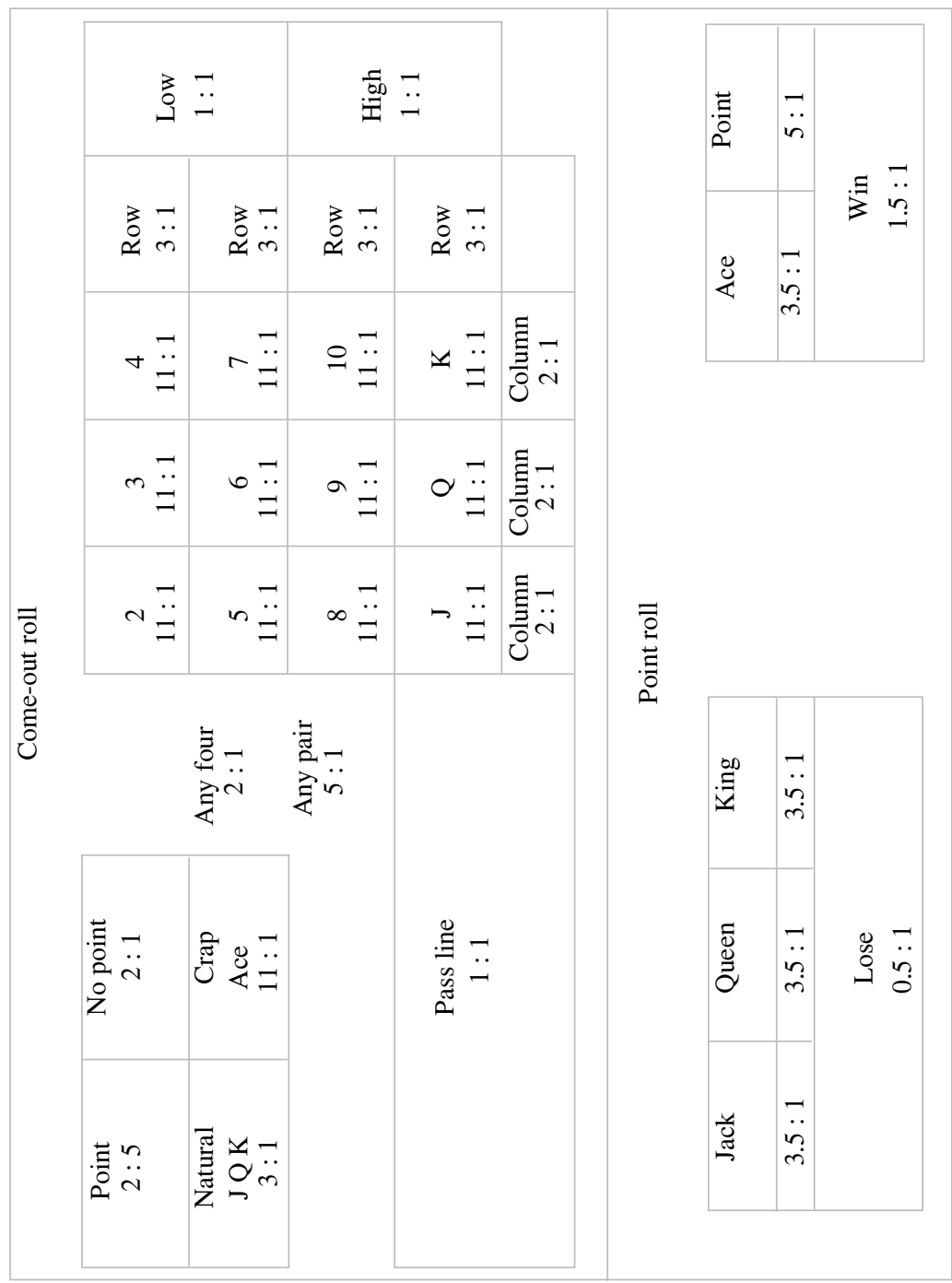

FIGURE 1: A betting table

\section{Length of the game}

In dice craps there is always an initial roll of two dice, and the game ends on one roll if the shooter rolls a sum of 7 or 11 or craps out with a sum of 2 , 3 , or 12 . So on average, dice craps ends on one roll 12 out of 36 times. In our game of Crapoulette, the game ends on one roll if the shooter draws a Jack, Queen, King, or Ace. So 4 out of 13 times, or about $30.77 \%$ of the time, the game ends on one draw. But what about the remainder of the time? 
Because rolling two dice always yields the same probabilities of outcomes, the number of additional rolls needed for dice craps can be analysed with the common geometric distribution. That is, if we have probability $p$ of success on each independent attempt, then the average number of attempts needed to succeed is $\frac{1}{p}$. So if a point of 4 is established, which occurs with probability $\frac{3}{36}$, then the shooter keeps rolling two dice until rolling a sum of 4 or 7 , which now occurs with probability $\frac{9}{36}=\frac{1}{4}$. In this case, it takes an average of 4 rolls to obtain a sum of 4 or 7 . It is the same for a point of 10 since initially rolling a 10 also occurs with probability $\frac{3}{36}$. So in the case of a point of 4 or 10 , which occurs with probability $\frac{3}{36}$, an average of 4 additional rolls is needed to complete a game of dice craps.

Similarly, for points of 5 or 9 , each of which occur with probability $\frac{4}{36}$, then the game ends when the shooter rolls the point or a 7 , which occurs with probability $\frac{10}{36}$. So in these cases, it takes an average of $\frac{36}{10}$ additional rolls to complete a game. Finally, for points of 6 or 8 , each of which occur with probability $\frac{5}{36}$, then the game ends when the shooter rolls the point or a 7 , which occurs with probability $\frac{11}{36}$. In these cases, it takes an average of $\frac{36}{11}$ additional rolls to complete a game. Having accounted for the additional rolls needed after the initial first roll, we obtain the average number of rolls in a game of dice craps to be

$$
\mathrm{E}[Y]=1+4 \times \frac{6}{36}+\frac{18}{5} \times \frac{8}{36}+\frac{36}{11} \times \frac{10}{36}=\frac{557}{165} \approx 3.3758
$$

In dice craps, the number of additional rolls needed depends on which point is established. However that is not the case in Crapoulette. In our game, each of the nine possible points occurs with probability $\frac{1}{13}$. If a point is established, then the shooter keeps drawing from the deck of 51 remaining cards, without replacement, until drawing one of 19 cards: a Jack, Queen, King, Ace, or another point. Because of non-replacement, the successive draws are no longer independent. In this case, the number of draws $Y$ needed to 'succeed' is a type of negative hypergeometric random variable: $Y \sim \operatorname{NGH}(51,19,1)$.

In general, the random variable $Y \sim \operatorname{NGH}(N, L, r)$ counts the number of draws needed to select $r$ of $L$ prespecified objects from a group of $N$ objects when randomly drawing without replacement [3]. The average number of draws needed is $\mathrm{E}[Y]=\frac{r(N+1)}{L+1}$. With this fact, we can derive the average length of our game.

Theorem 2: In Crapoulette, the average number of draws needed to complete a game is 2.8 .

Proof: We let $X$ be the total number of draws needed to complete a game, and let $Y$ be just the number of draws after the come-out roll in the event that a point is established. Then $Y \sim \operatorname{NGH}(51,19,1)$ and $\mathrm{E}[Y]=\frac{52}{20}$. Thus, by the Law of Total Expectation we have 


$$
\begin{aligned}
\mathrm{E}[X] & =\mathrm{E}[X \mid X=1] \mathrm{P}(X=1)+\mathrm{E}[X \mid X>1] \mathrm{P}(X>1) \\
& =1 \times \frac{4}{13}+(1+\mathrm{E}(Y)) \times \frac{9}{13}=1+\frac{52}{20} \times \frac{9}{13}=2.8 .
\end{aligned}
$$

\section{Conditional averages}

Once a point is established, the average number of remaining draws is $\mathrm{E}(Y)=\frac{52}{20}=2.6$. Interestingly enough, this average number of point rolls is independent of whether or not the shooter wins. That is, if we consider just the games won by the shooter after a point is established, then the average number of point rolls is 2.6. And among games lost by the shooter after a point is established, the average number of additional rolls is also 2.6. On the other hand, the overall average number of rolls for the entire game does depend on whether or not the shooter wins the pass line bet. To see these results, we shall use the probability distribution functions of $Y$ and $X$.

For $Y \sim \operatorname{NGH}(N, L, r)$, in order to take exactly $k$ draws to obtain one of the $L$ pre-specified objects, we first must have a permutation of $k-1$ objects from the other $N-L$ objects, followed by one of the $L$ objects. The probability of this event happening is then

$$
\mathrm{P}(Y=k)=\frac{\mathrm{P}(N-L, k-1) \times L}{\mathrm{P}(N, k)},
$$

for $1 \leqslant k \leqslant N-L+1$. Thus, the expected value of $Y$ is

$$
\mathrm{E}[Y]=\sum_{k=1}^{N-L+1} k \times \frac{\mathrm{P}(N-L, k-1) \times L}{\mathrm{P}(N, k)}=\frac{N+1}{L+1} .
$$

Now suppose the set of $L$ objects is further partitioned into two sets of $A$ elements and $B$ elements. In the case of Crapoulette, we have $L=19$ cards that end a game once a point is established. These 19 cards have $A=7$ winning cards and $B=12$ losing cards for the shooter. Now we consider only those games that end with one of the $A$ objects being chosen first, which we label as event $W_{1}$. In our case, $\mathrm{P}\left(W_{1}\right)=\mathrm{P}(W \mid X>1)=\frac{7}{19}$, as derived before Theorem 1. In general, $\mathrm{P}\left(W_{1}\right)=\frac{A}{L}$. Now given event $W_{1}$, the average number of point draws needed is

$$
\begin{aligned}
\mathrm{E}\left[Y \mid W_{1}\right] & =\sum_{k=1}^{N-L+1} k \times \mathrm{P}\left(Y=k \mid W_{1}\right)=\sum_{k=1}^{N-L+1} k \times \frac{\mathrm{P}\left(Y=k \cap W_{1}\right)}{\mathrm{P}\left(W_{1}\right)} \\
& =\frac{L}{A} \sum_{k=1}^{N-L+1} k \times \frac{\mathrm{P}(N-L, k-1) \times A}{\mathrm{P}(N, k)}=\mathrm{E}[Y] .
\end{aligned}
$$

Similarly, $\mathrm{E}\left[Y \mid W_{1}^{C}\right]=\mathrm{E}[Y]$, where $W_{1}^{C}$ is the complement of the event $W_{1}$, i.e. that the event $W_{1}$ does not occur.

For one entire game of Crapoulette, if a point is established then there can be at most 33 more rolls for a game-ending roll to occur, and the probability function for the total number of rolls needed is 


$$
\mathrm{P}(X=k)=\left\{\begin{array}{cl}
\frac{4}{13} & \text { if } k=1 \\
\frac{9}{13} \times \mathrm{P}(Y=k-1) & \text { if } 2 \leqslant k \leqslant 34,
\end{array}\right.
$$

where $Y \sim \operatorname{NGH}(51,19,1)$. From (1) we have $\mathrm{P}(X=1 \cap W)=\frac{3}{13}$. Also $\mathrm{P}(X=k \cap W)=\frac{9}{13} \mathrm{P}\left((Y=k-1) \cap W_{1}\right)$ for $2 \leqslant k \leqslant 34$. Now using (2) and (3), we find that in games that the shooter wins in Crapoulette, the average number of rolls is given by

$$
\begin{aligned}
\mathrm{E}[X \mid W] & =\sum_{k=1}^{34} k \mathrm{P}(X=k \mid W)=\sum_{k=1}^{34} k \frac{\mathrm{P}(X=k \cap W)}{\mathrm{P}(W)} \\
& =\frac{3 / 13}{\mathrm{P}(W)}+\frac{9 / 13}{\mathrm{P}(W)} \sum_{k=2}^{34} k \mathrm{P}\left((Y=k-1) \cap W_{1}\right) \\
& =\frac{3 / 13}{\mathrm{P}(W)}+\frac{9 / 13}{\mathrm{P}(W)} \sum_{k=1}^{33}(k+1) \mathrm{P}\left(Y=k \cap W_{1}\right) \\
& =\frac{3 / 13}{\mathrm{P}(W)}+\frac{9 / 13}{\mathrm{P}(W)}\left(\sum_{k=1}^{33} k \mathrm{P}\left(Y=k \cap W_{1}\right)+\sum_{k=1}^{33} \mathrm{P}\left(Y=k \cap W_{1}\right)\right) \\
& =\frac{3 / 13}{\mathrm{P}(W)}+\frac{9 / 13}{\mathrm{P}(W)}\left(\mathrm{E}[Y] \mathrm{P}\left(W_{1}\right)+\mathrm{P}\left(W_{1}\right)\right) \\
& =\frac{3 / 13}{120 / 247}+\frac{9 / 13}{120 / 247}\left(\frac{52}{20} \times \frac{7}{19}+\frac{7}{19}\right)=\frac{473}{200}=2.365 .
\end{aligned}
$$

An analogous argument can be used to compute the average number of rolls in those games lost by the shooter. Using $\mathrm{P}\left(W^{C}\right)=127 / 247$, $\mathrm{P}\left(W_{1}^{C}\right)=12 / 19, \mathrm{P}\left(X=1 \cap W_{1}^{C}\right)=\frac{1}{13}$ and $\mathrm{E}\left[Y \mid W_{1}^{C}\right]=\mathrm{E}[Y]$, we obtain $\mathrm{E}\left[X \mid W^{C}\right]=2039 / 635 \approx 3.211$. But we can also obtain this result using the Law of Total Expectation since

$$
\mathrm{E}[X \mid W] \mathrm{P}(W)+\mathrm{E}\left[X \mid W^{C}\right] \mathrm{P}\left(W^{C}\right)=2.8 \text {. }
$$

We leave it as an exercise to determine the conditional average number of rolls in dice craps among just winning games and losing games, and to determine whether or not the average number of additional rolls once a point is established depends on the shooter winning or losing.

\section{References}

1. Nevada State Gaming Control Board (2013), available at http://gaming.nv.gov/index.aspx?page=247

2. G. Grimmett and D. Welsh, Probability: an introduction, (2nd edn.) Oxford University Press, Oxford, (2014).

3. Norman L. Johnson, Adrienne W. Kemp, Samuel Kotz, Univariate discrete distributions, (3rd edn.), Wiley-Interscience (2005). 
doi:10.1017/mag.2015.26

DAVID K. NEAL and DUSTIN GENTILE

Department of Mathematics, Western Kentucky University, Bowling Green, KY 42101 USA e-mail:david.neal@wku.edu,dustin.gentile273@topper.wku.edu

\section{Announcement}

I am sorry to inform readers the deaths of two long-standing contributors to The Mathematical Gazette.

Llewellyn G Chambers, from Bangor, contributed notes and articles, largely on statistics, between 1983 and 2006, wrote book reviews and acted as a referee.

Robert J Clarke, who lived in Stourbridge, wrote on a wide variety of topics including algebra, analysis, number theory and geometry between 1996 and 2008, and won the Note of the Year Award in 1998.

Nemo

The answers to the Nemo page from March on arithmetic were:

1. E.J.Pratt

2. A.E. Housman

3. Charlotte Brontë

4. William Shakespeare

5. Emily Dickinson

6. Arthur Symons
The Truant

Fragment of a Greek Tragedy

Jane Eyre Chapter 13

Romeo and Juliet

Act 3, Scene 1

The Days that we can Spare

The Sick Man to Health

Congratulations to Henry Ricardo for identifying all these quotations. This month we turn the focus onto poems about spirals. (See page 212.) 